\title{
Flexible Link Arm with a Passive Gravity Compensation Mechanism: Design and Modelling
}

\author{
Juan Carlos Cambera ${ }^{(1)}$, Daniel Feliu-Talegon ${ }^{(1)}$, Saddam Gharab ${ }^{(1)}$, Vicente Feliu-Batlle ${ }^{(2)}$ \\ ${ }^{1}$ Instituto de Investigaciones Energéticas y Aplicaciones Industriales, University of Castilla-La Mancha. \\ ${ }^{2}$ Escuela Técnica Superior de Ingenieros Industriales, University of Castilla-La Mancha. \\ Emails: JuanCarlos.Cambera@alu.uclm.es, Daniel.Feliu@uclm.es, Saddam.Gharab@alu.uclm.es,Vicente.Feliu@uclm.es.
}

\begin{abstract}
The gravity compensation of flexible link manipulators using mechanisms based on counterweights has been previously studied in the research literature. However, as far as we are concerned, no attempt has been done to study the difficulties and benefits of constructing mechanisms for the same purposes based on springs. This paper presents a gravitational mechanism based on springs for a single-link flexible arm. In specific, we address the mechanical design of the mechanism and the dynamic modeling of the resulting system. To assess the effectiveness of the mechanism and the validity of the dynamic model, we present experimental results.
\end{abstract}

\section{INTRODUCTION}

The flexible link manipulators have certain properties that represent important advantages with regard to the more classical rigid manipulators. They are characterized by having lower energy consumption, reduced torque requirements, in addition to being lighter, cheaper and safer for human-robot cooperative tasks. These benefits are especially favorable for mobile robotic applications, in which there exist critical limitations of weight and energy. Depending on the characteristics of the environment (e.g., the presence of wind), the nature of the tasks and the mechanical design of the manipulator, the flexible links forming the manipulator can be subjected to external forces. The Group of Robotics and Automation at the University of Castilla-La Mancha is pursuing the development of robotics platforms with these characteristics for purposes of inspection and manipulation tasks. Up to the moment, two research projects funded by the Spanish Ministry of Economy and Competitiveness have structured around this direction. The first project is entitled "Development and Comparison of New Control Techniques for Very Large Lightweight Flexible Arms Mounted on Mobile Platforms Under Environmental Disturbances" (DPI2012-37062-CO201), the second project complements some aspect of the first one and is entitled "Control of Flex- ible Robots Under External Forces. Application to Force Sensors and Systems for Robot Positioning and Propulsion in Fluids" (DPI2016-80547$\mathrm{R})$. This paper presents an overview of the mechanical design and dynamic modeling of a single link flexible link arm with a passive gravity compensation mechanism based on linear springs. As will be seen in the following sections, the effect of the gravity compensation mechanism will be studied as an external force acting on an intermediate point of the flexible link. The overall system considered in this paper represents the first-degree-offreedom of the flexible link manipulator to be onboard of the mobile robot in the aforementioned project.

An important part of the torque requirements of a robot is used to counteract the effects of the force of gravity acting on the structure of the robot. The gravity compensation mechanisms are designed to cancel, at least partially, these effects on the actuators. This opens up the possibility of considering less powerful and, therefore, lighter and cheaper actuators, and also provides the option of improving the payload capacity and the speed of execution of the tasks. The design of passive gravity compensation mechanism falls normally into two categories: those based on counterweights, and the ones based on springs. In the counterweight category, there exists a constant exchange of gravitation potential energy between the mass of the robot and the counterweights, so that in the case of perfectly balanced manipulator the overall potential energy of the system remains constant, and no additional energy is required from the actuators. Although with these mechanisms the torque requirements of the manipulator are significantly reduced in the static conditions, the added inertia normally translates into larger dynamic torques during the execution of fast trajectories, not to mention an increment of the overall weight of the platform. Some important studies referred to the development of these mechanisms are found in $[2,5]$. The autonomous mobile robot called Gryphon, presented in [8], considers a manipulator, with a certain degree of link flexibility and gravity compensation based on counterweights, developed to assist in 
the task of demining. The manipulator consists of 3 degree-of-freedom configured in a pantographic form and with a counterweight that corresponds to the batteries of the system. The pantographic configuration of the arm permits the reallocate the motor of the manipulator near to its base, and with this contributes toward reducing the dynamic torque requirements of the system. In the case of spring based gravity mechanism, the condition of constant potential energy is achieved by the exchange of potential energy between the structure of the robot and the elastic potential energy stored in the springs. Unlike the previous case, the spring based mechanisms do not add significant inertia to the system, and therefore do not affect the dynamic torque requirements. However, these mechanisms pose serious mechanical design challenges owing to the gravitational torque is pose-dependent and non-linear by nature. The most known design principles take into account linear commercial springs disposed on geometric configurations that produce non-linear compensation torques by altering the length of the moment arm for each pose of the robot. Some of the most relevant work in this field can be found in $[10,13,9,12,11,1,6]$. Despite the attributes of the spring based gravity compensation, aside from own our research work, we have found no studies referred to its application to flexible link robots.

In this paper, we present our last advances in the development of a flexible link arm with a gravity compensation mechanism based on springs. In this paper, we will make special emphasis on the mechanical design: the decisions that lead us to the current prototype, and the description of the parts of the platform. At the end of the paper, we will also briefly present a dynamic model for the platform based on the lumped mass approach. It will come accompanied by experimental validation of the compensation mechanism and the dynamic model. A previous description of the dynamic model was carried out in [3]. We will extend the experimental validation of the previous paper to study the accuracy of the model when motor tracks a fast trajectory.

The paper has been organized in the following way. Section 2 gives a brief overview of the design and construction of the experimental platform. Section 3 presents the dynamic model. Section 4 presents the experimental validation of the mechanism and the dynamic model. Finally, in Sections 5 and 6, some concluding remarks and acknowledgment are stated, respectively.

\section{PLATFORM DESCRIPTION}

The development of a gravity compensation mechanism for a single link flexible arm starts from the development of the same type of mechanism for a single link rigid arm. In the case of the latter, the simplest mechanism is described in the diagram presented in Fig. 1. In this mechanism, two conditions need to be satisfied in order to achieve the perfect gravity compensation. These can be easily obtained from studying the settings that permit that the sum of all the torques about the motor axis is equal to zero in static conditions: (1) the spring constant must have a value of $k_{\text {spring }}=\frac{\left(\frac{m_{l i n k}}{2}+m_{t i p}\right) g l}{l_{s}{ }^{2}}$ (the nomenclature is detailed in Section 3); (2) the distance between the motor axis and the points of attachment of the spring (points $\mathrm{A}$ and $\mathrm{B}$ ) must be equal. The latter condition is not physically possible since it implies that the natural length of the spring must be equal to zero. This difficulty could be resolved by considering the alternative design presented in the same figure, Fig. 1, which counts exactly with the same mechanical properties than the previous one (see other alternatives in [11]). Since only a very limited number of springs are commercially available, the designer needs to adjust to the distance of attachment of the spring to the rigid arm, and also consider the possibility of using several springs in parallel to meet the requirements of the elasticity constant.

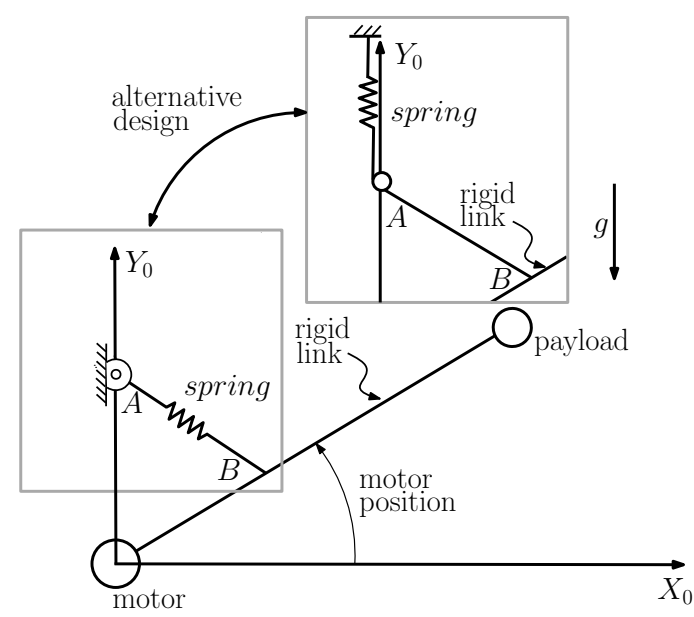

Figure 1: Gravity compensation system for one link rigid link.

The gravity compensation mechanism we propose for the case of a single-link flexible arm is the implementation of the alternative design, commented in the previous paragraph, for the case of a single-link rigid arm. The exact gravity compensation is not attainable in the case of the flexible arm, at least with the proposed design. However, taking into account that the flexible link consid- 
ered only exhibits small deflections, very significant reductions in the motor torque requirements are attainable. The photograph of the experimental platform of the single link flexible arm with the gravity compensation mechanism is showed in Fig. 2. It is composed by a brushless DC motor coupled to a zero backlash gearbox, a flexible link made of composite materials with a hollow tubular cross section, a plastic structure used to retain the payload mass at the tip of the flexible arm, and the actual gravity compensation mechanism, which will be explained in the following paragraph. As sensors, we considered an incremental encoder to measure the motor position, and a two set of strain gauges to measure the torque at the base and at an intermediate point of the link. A National Instrument PXI system is used to capture the sensor measurements, run the control algorithms, and finally command the motor through a servo drive. A motion capture system is used to validated the performance of the system by measuring the tip position of the arm at a sampling rating of $100 \mathrm{~Hz}$ with a standard deviation of $1 \mathrm{~mm}$.

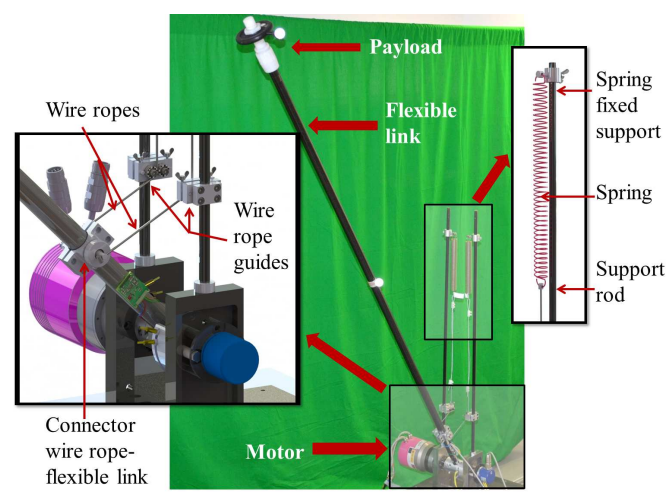

Figure 2: Photograph of the single link flexible robot with the passive gravity compensation mechanism.

From Fig. 2 can be observed that the gravity compensation mechanism was symmetrically built at both sides of the plane of movement of the flexible arm. This design decision allowed us to guarantee the proper balance of the platform. The compensation mechanism is formed mainly by six type of pieces. For sake of simplicity, only one side of the compensation mechanism will be explained. A supporting rod (part type 1) is vertically arranged and fixed to the structure that supports the body of the motor. As can be seen in Fig 3, in the upper part of the rod, a fixed support (part type 2) provides a point of attachment to a linear extension spring (parts type 3 ). The lower part of the spring is attached to a wire rope (parts type 4), which maintains a vertical orientation until crossed the wire rope guide (parts type
5). The function of the latter piece is to redirect the wire rope to the link, where it is connected to the attachment connector (parts type 6). The wire rope guide is formed by two rollers arranged side by side that change the direction of the wire rope with minimum friction and, at the same time, maintain the wire rope entrapped for any configuration of the arm. A detailed view of the wire rope guide is shown in Fig 4 . The attachment connector is used to fasten the wire rope to the flexible link without requiring drilling operations and guaranteeing the automatic alignment of the wire rope with the lower part of the lower part of the wire rope guides. Avoiding the drilling of the flexible link made of composite material is important to avoid the delamination of the material, which would change the mechanical properties of the link. The automatic alignment of the wire rope is vital in order to avoid the accumulation of unnecessary tension in the springs that would lead to incorrect gravity compensation. The automatic alignment of the wire rope is achieved by connecting the wire rope to an axis mounted on ball bearing, which permits its free rotation. A detailed view of the attachment connector is shown in Fig 5.

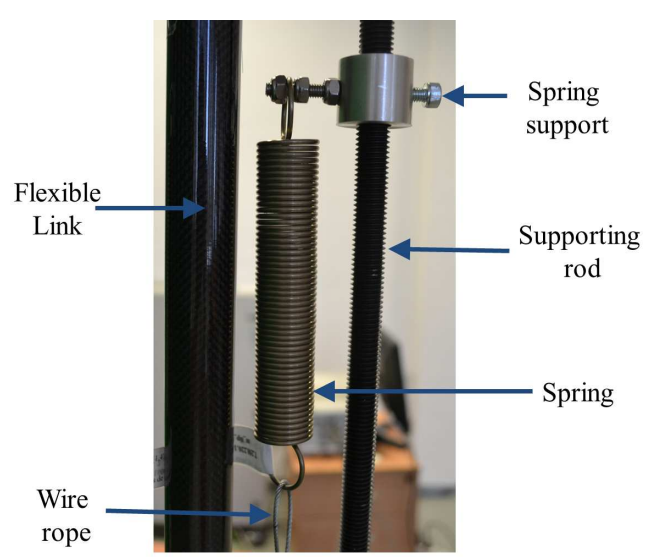

Figure 3: Photograph of the upper part of the supporting rods.

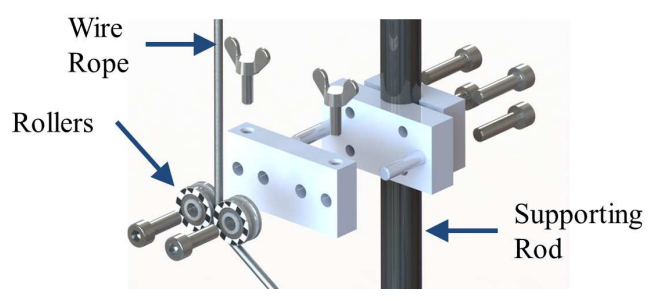

Figure 4: CAD exploded view of the wire rope guide.

Table 1 provides the values of the main parameters of the experimental platform. The gravity compensation mechanism was configured to coun- 


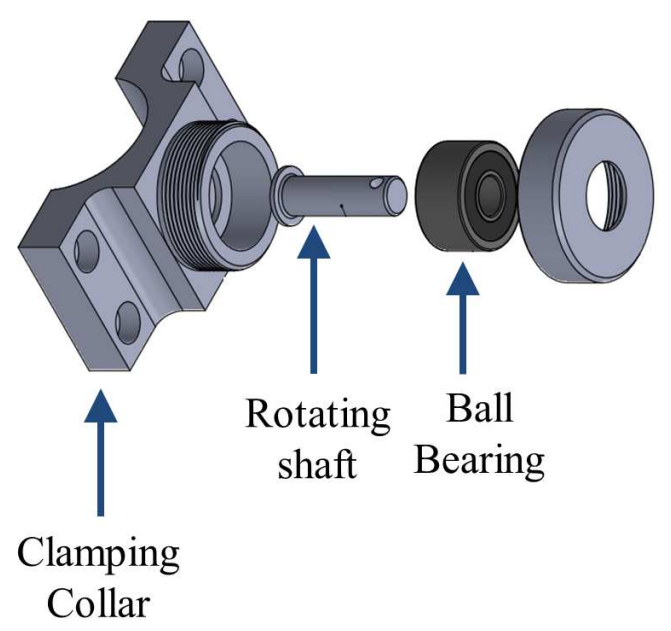

Figure 5: CAD exploded view of the attachment connector.

teract the gravitational torque when the flexible link arm supports the payload $m_{t i p-2}$. The other two payload masses presented in the table, $m_{t i p-1}$ and $m_{t i p-3}$, were used for identification and validation purposes.

\section{DYNAMIC MODEL}

The dynamic model of the system is presented as two interconnected subsystems: a DC motor subsystem and the flexible link arm. The variable that interrelates these subsystems is the coupling torque, which represents the torque that the motor exerts on the base of the flexible link.

The dynamics of the DC motor is given by

$$
\Gamma_{m}=K_{m} n_{r} i_{m}=J \ddot{\theta}_{m}+\nu \dot{\theta}_{m}+\Gamma_{\text {st-coul }}+\Gamma_{\text {coup }},
$$

It is assumed that the armature current of the motor $i_{m}$ can be instantaneously configured with the servo drive. Therefore, the dynamics of the electrical part of the motor is considered much faster than the mechanical part. In the previous equation, $\theta_{m}$ is the motor position, $n_{r}$ is the gear ratio, $J$ is the rotor inertia, $\nu$ is the coefficient of viscous friction, $\Gamma_{s t-c o u l}$ represents the static and Coulomb frictions, and $\Gamma_{\text {coup }}$ is the coupling torque.

The flexible link arm with the gravity compensation mechanism was dynamically modeled starting from the lumped mass modeling methodology presented in [7]. In the lumped mass approach, the flexible arms are modeled as a set of point-

\footnotetext{
${ }^{1}$ The parameters experimentally identified are indicated with a $(*)$.
}

Table 1: Parameters of the system. ${ }^{1}$

\begin{tabular}{|c|c|c|}
\hline Description & Symbol & Value \\
\hline \multicolumn{3}{|c|}{ Actuator Parameters } \\
\hline Motor model & - & $\begin{array}{c}\text { Harmonic Drive } \\
\text { CHA-25A-100-D-SP }\end{array}$ \\
\hline Gear ratio & $n_{r}$ & 100 \\
\hline Motor maximum torque & - & 157 N.m \\
\hline Motor continuous stall torque & - & 108 N.m \\
\hline Motor torque constant & $K_{m}$ & $7.410^{-3}$ N.m/A \\
\hline Motor rotor inertia $(*)$ & $J$ & $6.19 \mathrm{Kg} \cdot \mathrm{m}^{2}$ \\
\hline Motor viscous friction( $\left.{ }^{*}\right)$ & $\nu$ & $2.16 \mathrm{Kg} \cdot \mathrm{m}^{2}$ \\
\hline Static friction $(*)$ & - & 18.18 N.m \\
\hline \multicolumn{3}{|c|}{ Link Parameters } \\
\hline Type of section & - & Tubular \\
\hline Link length & $l$ & $2.02 \mathrm{~m}$ \\
\hline Link mass & - & $0.27 \mathrm{Kg}$ \\
\hline Flexural rigidity $(*)$ & $E I_{z}$ & $841.78 \mathrm{~N} \mathrm{~m}^{2}$ \\
\hline Link gravitational mass $1\left(^{*}\right)$ & $m_{\lg 1}$ & $0.137 \mathrm{Kg}$ \\
\hline Link inertial mass $1(*)$ & $m_{l a 1}$ & $0.117 \mathrm{Kg}$ \\
\hline Link gravitational mass $2(*)$ & $m_{\lg 2}$ & $0.080 \mathrm{Kg}$ \\
\hline Link inertial mass $2\left({ }^{*}\right)$ & $m_{l a 2}$ & $0.032 \mathrm{Kg}$ \\
\hline Payload mass 1 & $m_{t i p-1}$ & $1.83 \mathrm{Kg}$ \\
\hline Payload mass 2 & $m_{t i p-2}$ & $2.13 \mathrm{Kg}$ \\
\hline Payload mass 3 & $m_{t i p-3}$ & $2.43 \mathrm{Kg}$ \\
\hline Link $1^{\text {st }}$ freq. for mass 1 & - & $2.01 \mathrm{~Hz}$ \\
\hline Link $1^{\text {st }}$ freq. for mass 2 & - & $1.87 \mathrm{~Hz}$ \\
\hline Link $1^{\text {st }}$ freq. for mass 3 & - & $1.75 \mathrm{~Hz}$ \\
\hline Strain to torque constant $1\left({ }^{*}\right)$ & $\overline{K_{g-1}}$ & $24.40 \mathrm{~N} \mathrm{~m} / \mathrm{V}$ \\
\hline Strain to torque constant $2\left({ }^{*}\right)$ & $K_{g-2}$ & $20.59 \mathrm{~N} \mathrm{~m} / \mathrm{V}$ \\
\hline \multicolumn{3}{|c|}{ Gravity Compensator Parameters } \\
\hline Spring constant $(*)$ & $K_{\text {spring }}$ & $597.80 \mathrm{~N} / \mathrm{m}$ \\
\hline Distance of attachment 1 & $l_{1}$ & $0.250 \mathrm{~m}$ \\
\hline Distance of attachment $2\left(^{*}\right)$ & $l_{s}$ & $0.297 \mathrm{~m}$ \\
\hline
\end{tabular}

masses interconnected by mass-less flexible elements. In our particular case, it was necessary to adapt the initial methodology to consider the movement of a flexible arm in a vertical plane and in presence of gravity force, and not only into a horizontal plane. In addition to that, the principles on the initial methodology stated that the number of point-masses is equal to the number of mass-less flexible elements, and this number defines the number of vibrational modes to be modeled. In the case of our approach, we relaxed this conditions considering two flexible elements with only a single lumped mass. The resulting model is capable of modelling the first vibrational mode of the flexible arm with the added capability of being able to consider the effect of an external force acting on an intermediate point of the link, and maintaining the property of minimum phase in the relationship between the motor position and the tip position of the arm. The minimum phase property of the model is very relevant from the point of view of the control algorithm design since it facilitates its design. The dynamic model of the flexible arm with the current gravity compensation mechanism was presented in [3]. In the following paragraph, we present a brief description of it. For more details refer to the previous paper.

Fig. 6 shows a schematic representation of the system. In order to simplify the discussion about the dynamic model, in the schematic representation, we presented a unique spring connecting the points A-B. In the previous section, we stated that it is an equivalent representation of the actual system, in which two springs in parallel are arranged 
out of the path between the points A and B.

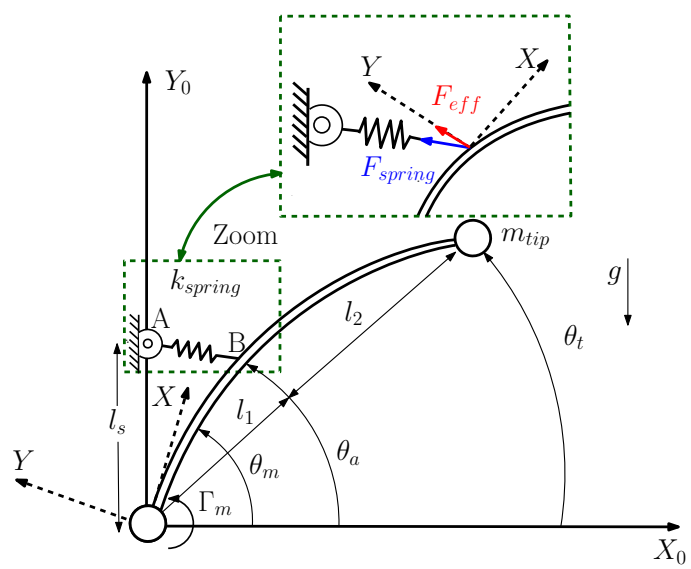

Figure 6: One degree of freedom gravity compensated flexible link robot described by a single lumped mass model.

The dynamic model of the flexible link subsystem is described by

$$
\gamma_{1} \ddot{\theta}_{t}+\gamma_{2} \cos \left(\theta_{t}\right)+l\left(\frac{\lambda^{3}-3 \lambda^{2}}{2}\right) F_{e f f}+\frac{3 E I_{z}}{l}\left(\theta_{t}-\theta_{m}\right)=0, \quad(2)
$$

where the constants $\gamma_{1}$ and $\gamma_{1}$ are given by

$\gamma_{1}=l^{2}\left(m_{t i p}+m_{l a 1}\left(\frac{3 \lambda-\lambda^{2}}{2}\right)+m_{l a 2}\left(\frac{2-3 \lambda+\lambda^{2}}{2}\right)\right)$,

$\gamma_{2}=\lg \left(m_{t i p}+m_{l g 1}\left(\frac{3 \lambda-\lambda^{2}}{2}\right)+m_{l g 2}\left(\frac{2-3 \lambda+\lambda^{2}}{2}\right)\right)$.

The variables of the model are the angular position of the point of the attachment of the spring $\left(\theta_{a}\right)$, the angular position of the tip $\left(\theta_{t}\right)$, and the effective force $\left(F_{\text {eff }}\right)$, which is the component of the force exerted by the spring $\left(F_{\text {spring }}\right)$ that actually affects the flexible link arm (see Fig. 6). The parameters are the payload mass $\left(m_{t i p}\right)$, the spring elasticity constant $\left(k_{\text {spring }}\right)$, the gravitational constant $(g)$, the flexural rigidity $\left(E I_{z}\right)$, the distance between the motor axis and the attachment point of the spring to the rigid support $\left(l_{s}\right)$, the total length of the flexible link arm $(l)$, which can be also expressed as the sum of the length of the two massless flexible elements $\left(l_{1}\right.$ and $\left.l_{2}\right)$. In the equations, and for the sake of a more compact notation, the distance between the motor axis and the attachment point of the spring to the flexible link $\left(l_{1}\right)$, is expressed in the form of $l_{1}=\lambda l$, where $\lambda$ is a proportionality constant between zero and one.

Assuming the hypothesis that the link only exhibits small deflections, the effective force $F_{\text {eff }}$ can be approximated in the following way

$$
\begin{aligned}
F_{\text {eff }} & =k_{\text {spring }}\left(\lambda l \sin \left(\theta_{m}-\theta_{a}\right)+l_{s} \cos \left(\theta_{m}\right)\right) \\
& \approx k_{\text {spring }}\left(\lambda l\left(\theta_{m}-\theta_{a}\right)+l_{s} \cos \left(\theta_{m}\right)\right),
\end{aligned}
$$

To make the dynamic model useful for the control, the variables $\theta_{t}, \theta_{a}$ and $F_{\text {eff }}$ need to be estimated from the measurements available in the system: the motor position $\left(\theta_{m}\right)$, the coupling torque $\left(\Gamma_{\text {coup }}\right)$, and the torque at the point of attachment of the spring on the link $\left(\Gamma_{a}\right)$. The motor is measured by using an encoder, whereas the torques are estimated by two strain gauges arrangements placed at the base and at the point of attachment of the spring. The estimations of the mentioned angles are given by

$$
\begin{aligned}
& \hat{\theta}_{a}=\theta_{m}-\frac{l \lambda}{6 E I_{z}}\left(\Gamma_{a}+2 \Gamma_{\text {coup }}\right), \\
& \hat{\theta}_{t}=\theta_{m}+\frac{l}{6 E I_{z}}\left((\lambda-2) \Gamma_{a}+\left(-3 \lambda+\lambda^{2}\right) \Gamma_{\text {coup }}\right) .
\end{aligned}
$$

The estimation of the effective force $\left(F_{\text {eff }}\right)$ can be obtained by two methods. The estimation on the first method does not depend on spring model and is given by

$$
\hat{F}_{\text {eff }}=\rho_{1} \cos \left(\hat{\theta}_{t}\right)-\frac{1}{l_{1}} \Gamma_{\text {coup }}+\rho_{2} \Gamma_{a},
$$

where $\rho_{1}$ and $\rho_{2}$ are

$$
\begin{aligned}
\rho_{1} & =\frac{m_{t i p} g}{\lambda\left(m_{t i p}+m_{l a 2}\right)}\left(m_{l a 2}-m_{l a 1}-m_{l g 2}+m_{l g 1}\right) \\
& +\frac{g}{\lambda\left(m_{t i p}+m_{l a 2}\right)}\left(m_{l a 2} m_{l g 1}-m_{l a 1} m_{l g 2}\right), \\
\rho_{2} & =\frac{\left(m_{t i p}+m_{l a 1}\right)}{l(1-\lambda)(\lambda)\left(m_{t i p}+m_{l a 2}\right)} .
\end{aligned}
$$

The estimation of the second method relies completely on the model of the spring, equation (5), and the estimation of the angular position of the attachment point, equation (7). It is given by

$$
\hat{F}_{\text {eff }}=k_{\text {spring }}\left(\lambda l \sin \left(\theta_{m}-\hat{\theta}_{a}\right)+l_{s} \cos \left(\theta_{m}\right)\right) \text {. }
$$

\section{EXPERIMENTAL VALIDATION}

The experimental validation is presented in two parts. The first part is concerned with evaluating the capability of the mechanism to cancel the gravitational torque at the output of the motor. The second part addresses the validation of the dynamic modeling. The parameters considered in the simulation of the dynamic model were obtained by following the identification methodology explained in [3]. 


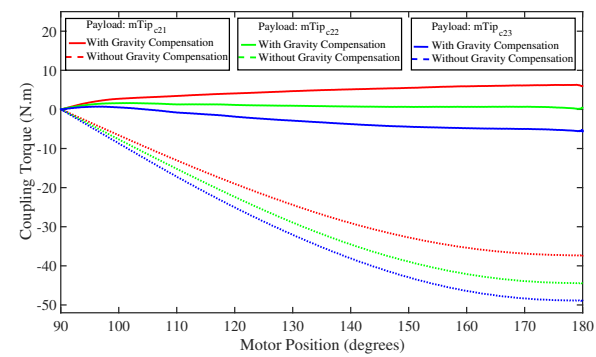

Figure 7: Comparison of the coupling torque for the system with and without gravity compensation in static conditions (experiment).

With regard to the evaluation of the capability of the gravitational mechanism to cancel the gravitational torque, Fig 7 compares the coupling torque for the system with and without gravity compensation in the case that different payload masses and motor orientations are considered. At this point, it is important to remember that gravity compensation mechanism was configured to cancel the vibration of the payload mass $m_{t i p-2}$. For that particular payload mass and considering the position of largest gravitational torque $\left(\theta_{m}\right.$ about 180 degrees), the torque reduction was about 44.06 N.m, representing a reduction of $98.4 \%$ of the gravitation torque requirement of the system without gravity compensation mechanism. Taking into consideration that the continuous stall torque of the motor 108 N.m, the torque reduction adds an extra $40 \%$ of continuous torque that could be used to improve the speed of the system.

With regard to the validation of the dynamic model, Figs. 8-10 show the comparison between the experimental data and simulated data for the tip position, coupling torque and attachment torque in the case in which the motor tracks a fourth order trajectories that goes from 180 to 135 degrees in 2 seconds. For these experiments, a motor position controller was used to track the trajectory, whereas no controller was used to cancel the vibration at the tip. The motor position controller corresponded to the two degrees of freedom controller detailed in [4]. With regard to the tip position, the model behaves very similarly to behavior than the experimental data with a fitness of $99.5 \%$, calculated as the Normalized Mean Square Error (NMSE). The maximum error between these plots is of about $6 \mathrm{~mm}$ for a flexible link of 2 meters in length. The coupling torque and the attachment torque were also reproduced by the model with a high level of precision, i.e., a fitness of $81.7 \%$ and $87.4 \%$ (NMSE), respectively.

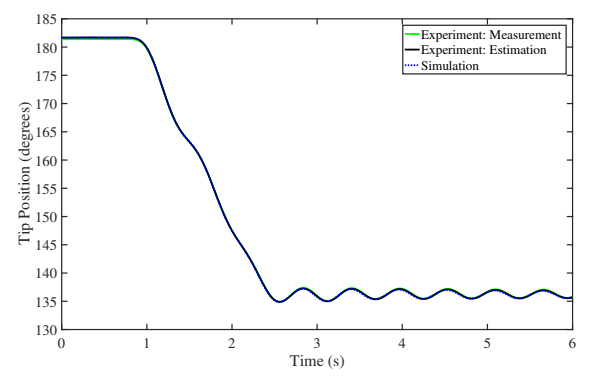

Figure 8: Tip position comparison (experiment vs. simulation)

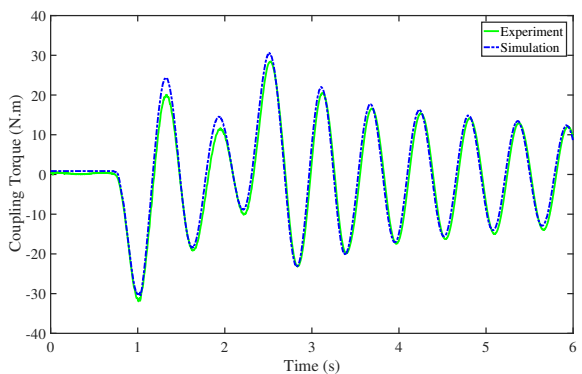

Figure 9: Coupling torque comparison (experiment vs. simulation).

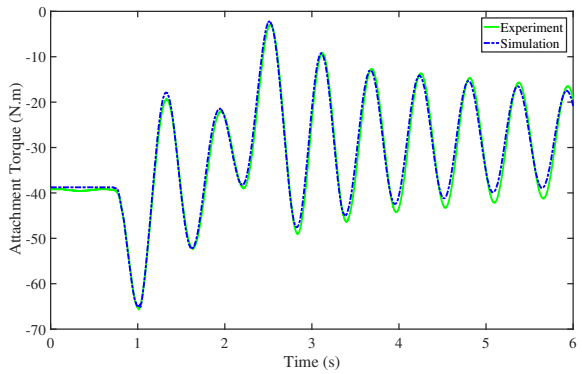

Figure 10: Attachment torque comparison (experiment vs. simulation). 


\section{CONCLUSIONS}

In this paper, we presented a gravity compensation mechanism based on springs for a single-link flexible arm. We experimentally validated the mechanism achieving a near perfect-gravity cancellation, which represents a $98.4 \%$ of the gravitational torque reduction in the more demanding case scenario,i.e., the motor position is about the horizontal orientation. During the paper, we explained in detailed the design considerations that lead to the current prototype, and the actual mechanical design that made possible gravity compensation. In addition to the previous, we also presented a dynamic model for the gravity compensated flexible arm based on the lumped mass approach. The development of the dynamic model started from the methodology presented by Feliu in [7] for the case of a single link flexible arm moving in the horizontal plane. For our purposes, we adapted the development presented there to incorporate the case of movements in a vertical plane and under the action of gravitational force, and to consider the effect of gravity compensation mechanism that was represented as an external force acting on an intermediate point of the flexible link. The resulting model describes the behavior of the first vibrational mode of the arm. The dynamic model was validated experimentally considering the case in which the motor tracked a fourth order trajectory that went from 180 degrees (horizontal orientation) to 135 degrees in 2 seconds, whereas no controller was considered to cancel the vibrations in the arm. In that case, the comparison between the experiment and the simulation showed an average fitness of 89.5\%(NMSE) for the signals compared. Future work will address the development of a controller for the tip positioning of the flexible arm.

\section{ACKNOWLEDGMENTS}

This work was supported by the Agencia Estatal de Investigación (AEI) of Spain under project DPI2016-80547-R (Ministerio de Economía y Competitividad) and co-financed by the European Social Fund (FEDER, EU).

\section{References}

[1] Agrawal, S. K., And Fattah, A. Gravitybalancing of spatial robotic manipulators. Mechanism and machine theory 39, 12 (2004), 1331-1344.

[2] Agrawal, S. K., Gardner, G., And PledgIE, S. Design and fabrication of an active gravity balanced planar mechanism using auxiliary parallelograms. Journal of mechanical design 123, 4 (2001), 525-528.

[3] Cambera, J. C., Chocoteco, J. A., And Feliu-Batlle, V. Modeling and identification of a single link flexible arm with a passive gravity compensation mechanism. In Robotics and Automation (ICRA), 2018 IEEE International Conference on (2018), IEEE.

[4] Cambera, J. C., And Feliu-Batlle, V. Input-state feedback linearization control of a single-link flexible robot arm moving under gravity and joint friction. Robotics and $A u$ tonomous Systems 88 (2017), 24-36.

[5] Chung, W.-K., And Cho, H. S. On the dynamic characteristics of a balance puma-760 robot. Industrial Electronics, IEEE Transactions on 35, 2 (1988), 222-230.

[6] Endo, G., Yamada, H., Yajima, A., Ogata, M., And Hirose, S. A passive weight compensation mechanism with a noncircular pulley and a spring. In Robotics and Automation (ICRA), 2010 IEEE International Conference on (2010), IEEE, pp. 38433848 .

[7] Feliu, V., Rattan, K., And Brown, H. Modeling and control of single-link flexible arms with lumped masses. Journal of $d y$ namic systems, measurement, and control 114, 1 (1992), 59-69.

[8] Freese, M., Matsuzawa, T., Oishi, Y., Debenest, P., Takita, K., Fukushima, E. F., And Hirose, S. Robotics-assisted demining with gryphon. Advanced Robotics 21, 15 (2007), 1763-1786.

[9] Gopalswamy, A., Gupta, P., And VidyASAGAR, M. A new parallelogram linkage configuration for gravity compensation using torsional springs. 664-669.

[10] Mahalingam, S., And Sharan, A. M. The optimal balancing of the robotic manipulators. 828-835.

[11] Morita, T., Kuribara, F., Shiozawa, Y., And Sugano, S. A novel mechanism design for gravity compensation in three dimensional space. In Institute of Electrical and Electronics Engineers Inc. (2003).

[12] Rahman, T., Ramanathan, R., SelikTAR, R., AND HarWin, W. A simple technique to passively gravity-balance articulated mechanisms. Transactions-American Society Of Mechanical Engineers Journal Of Mechanical Design 117 (1995), 655-657. 
[13] Ulrich, N., and Kumar, V. Passive mechanical gravity compensation for robot manipulators. 1536-1541.

(C) $\$$ C) 2018 by the authors.
Submitted for possible
open access publication (http://creativecommons.org/licenses/by-nc/3.0/). 